\title{
Index autorum ad Vol. 12
}

Adler, P. 366 Adler-Hradecky, C. 366 Åkesson, H. O. 28 Balakrishnan, V. 322 Beckman, L. 20, 117 Beer, H. 103

Bellefeuille, P. de 123, 378 Bernini, L. 202 Book, J. A. 193 Bresler, J. B. 359 Choaripour, R. 45

Colucci, C. F. 202 Defrise-Gussenhoven, E. 65 Dubiska, A. 136 Dubiski, S. 136 Eftekhari,M. 45 Elston, R. 117 Eriksson, A. 242, 312 Fleischer, E. A. 281 Forsius, H. 230 Freire-Maia, N. 97, 209, 212 Frisk, M.D. 312 Grumbach, A. 103 Gustavson, K.-H. 20, 156 Hadley, H. G. 178 Heiken, A. 352 Huizinga, J. 1 Klunker, W. 56 Krieger, H. 97, 212

Lehmann, W. 230 Leonhardt, T. 251 Levy, J. 33 Lundin, L.-G. 156 Marçallo, F. A. 212 Margolis, E. 12 Masterson, J. G. 193, 219 Matsuda, E. 262 Matsunaga, E. 262 Melchior, J. C. 164 Michele, D. de 202 Mikkelsen, M. 164 Mohr, J. 51, 281 Murai, K. 262 Nevanlinna, H. R. 312 Norring, A. 20 Okajima, M. 172 Pelt, J. F. van 1 Persson, I. 41, 292 Piomelli, S. 202 Rapacz, J. 136 Reinskou, T. 51 Rivat, L. 45 Ropartz, C. 45 Rousseau, P.-Y. 45 Salzano, F. M. 212 Santesson, B. 193 Siniscalco, M. 202 Walter, H. 296 